\title{
Tungusische Lehnwörter im Ostjakischen
}

1. Die Erörterung der Frage etwaiger gemeinsamer Schichten im Wortschatz des Ostjakischen und tungusischer Sprachen hat bisher im weitgesteckten Rahmen der Diskussion über die uralaltaische Urverwandtschaft stattgefunden. In Arbeiten von A. Saurageot oder M. Räsänen beispielweise finden sich neben Angaben aus zahlreichen uralischen und altaischen Sprachen auch etliche tungusisch-ostjakische Wortvergleichungen. Der Anhang "Uralo-Altaica" im B. Collinders Fenno-Ugric Vocabulary enthält ebenfalls eine Anzahl derartiger etymologischer Hinweise. ${ }^{1}$

Dem Problem, dass der ostjakische Wortschatz ausserdem noch jüngere tungusische Elemente zu enthalten scheint, wurde m.W. bisher nicht systematisch nachgegangen. Der Aufdeckung tungusischer Lehnwörter im Ostjakischen stand vermutlich hemmend die Annahme im Wege, nach der die Ostjaken während der Zeit ihrer sprachlicher Eigenständigkeit keine nenneswerten Kontakte zu Tungusen gehabt haben. ${ }^{2}$

I Auf die Frage der Lralo-Altaica kam Collinder in seinem Vortrag am Zweiten Internationalen Finnougristenkongress (1965 in Helsinki) zurück: "In meinem 'Fenno-Lgric Vocabulary' habe ich zweiundsiebzig sorgfältig ausgewählte Zusammenstellungen angeführt, auf drei Gruppen verteilt. Die erste Gruppe enthält Wörter, die uralischerseits auch im Samojedischen vorkommen. In der zweiten Gruppe findet man Wörter die auf das Gemeinfinnischugrische, aber nicht auf das Gemeinuralische zurückgeführt werden können. Die dritte Gruppe umfasst Wörter die sogar nicht als gemeinfinnischugrische angesetzt werden dürfen. Vorausgesetzt dass wir es hier mit Entlehnungen aus altaischen Sprachen zu tun haben, wāre wohl zu erwarten, dass die dritte Gruppe die grösste sei. ... In Wirklichkeit gibt es in der ersten Gruppe zweiundfünfzig Wörter, in der zweiten siebzehn, in der dritten drei.» (Hat das Uralische Verwandte? [vervielfältigtes Manuskript], S. 23.)

2 Schon 1963 regte D. Sinor die Prüfung etwaiger mongolischer und 
Damit die Diskussion über das angedeutete Problem beginnen kann, möchte ich im vorliegenden Beitrag 1) anhand einiger Beispiele zeigen, dass es im Ostjakischen Wörter gibt, die sehr wahrscheinlich unter Ausschluss einer Vermittlersprache, wie z.B. Samojedisch, aus tungusischen Dialekten entlehnt worden sind, und 2) kurz die Frage der unmittelbaren tungusischostjakischen Beziehungen berühren. Späteren Untersuchungen sei die ausführliche Behandlung des Fragekomplexes vorbehalten. Als Beispiele habe ich solche Wörter gewählt, die in der 1. Lieferung (1966) des "Dialektologischen und etymologischen Wörterbuchs der ostjakischen Sprache» von W. Steinitz (im weiteren DEW) ohne Etymologie bleiben.

2. Das erste Beispiel ist öladk (Trj.), Mj. ŏlajk-, Kaz. ălak-, $\mathrm{O}$ ălàk, Vol. ásar 'Halsgeschirr des Renntiers (O); Zuggeschirr des Renntiers (Trj.)' (DEW 91). Zu Karjalainens Vermutung "vielleicht samojedischen Ursprungs" (Zur ostjakischen Lautgeschichte 188) stellt Steinitz nachdrücklich fest: »ein samoj. Original existiert nicht). (Auch Karjalainen teilte mit, dass ihm ein etwaiges samojedisches Original nicht bekannt sei.)

Das Original des ostjakischen Wortes scheint folgendes zu sein: Evenkisch (im weiteren: ev.) alag 'Zuggeschirr (für Rentiere oder Hunde); Tragriemen, Schleppseil' (J. Benzing, Die tungusischen Sprachen, Wiesbaden 1955, 20; G. M. Vasilevič, Évenkijsko-russkij slovar', Moskva 1958, 22; im weiteren: Benz. bzw. Vas.)

Dass die Entlehnungsrichtung nur Evenkisch $\rightarrow$ Ostjakisch sein kann, zeigt schon die weite Verbreitung des Wortes bzw. Wortstammes im ganzen tungusischen Sprachgebiet: Nanaisch, Negidalisch, Orotschisch, Udiheisch ala, Lamutisch al (Benz. 20; Vas. 22). Im Evenkischen kommt ausserdem auch ala vor (Vas. 22), ebenso alig bzw. alik (Vas. 25). Benzing setzt für das Wort eine urtung. Form *xala an (20). Ausserdem kann es aber schon in urtung. Zeit die Form *xalag gegeben haben, da das Suffix *-g bereits fürs Urtung. nachweisbar ist (vgl. Benz. 68 f.). ${ }^{1}$

tungusischer Elemente im Wortschatz der ugrischen Sprachen an (Introduction à l'Etude de l'Eurasie Centrale, S. 12).

1 Für freundliche Beratung in Fragen der Altaistik danke ich Herrn Prof. G. Doerfer (Göttingen). 
Da die Ostjaken die Rentierkultur erst nach dem 14. Jahrhundert von ihren Nachbarn - wie man bisher annahm, ausschliesslich von Juraksamojeden (vgl. P. Hajdú, Finnugor népek és nyelvek 178, 344; id.: The Samoyed Peoples and Languages 11) - übernommen hatten, hat das evenkische Wort sicherlich nach dieser Zeit Eingang ins Ostjakische gefunden. Auch die nur regionale Verbreitung des Wortes bei den Ostjaken legt die Annahme einer verhältnismässig jungen Entlehnung nahe (auch die Vach-Ostjaken benutzen das Rentier als Zugtier!, doch das Wort ist nicht mehr zu ihnen gelangt).

Der Anlaut weist auf Nordostjaken als unmittelbare Entlehner hin. Wenn aber Surg. ŏ sekundär ist, und das muss es wegen der klaren Entsprechung ev. $a$ : ostjN $\breve{a}$ sein ${ }^{1}$, kann die im Anlaut der ostjakischen Formen zutage tretende Konstellation nicht auf das Urostjakische zurückgeführt werden - ein weiteres Argument für die jüngere Entlehnungszeit. Dic übrigen Lautentsprechungen sind regelmässig ${ }^{2}$, die Substituierung des auslautenden $g$ durch $k$ ist auch erwartungsgemäss (vgl. Y. H. Toivonen, U̇ber die syrjänischen Lehnwörter im Ostjakischen, FUF XXXII, insbesondere 107 f.).

3. Das nächste Beispielwort ist olax (KoP Kr.; folk.), NiSt. -əlax, Patk. (K) ōlax, Reg. ălăch, Páp. ảlax 'Schlauheit (Kr.); Weisheit, Kunstgriff (Patk.); - KoP pila xuj pitat mătamtat olxə $\eta x$ uj olxat $m$. [er zeigt die Zauberkunst des zauberkundigen Mannes, die Schlauheit des schlauen Mannes]' (DEW 91).

Als sein Original kann angesehen werden ev. uläk 'Lüge, Betrug, Täuschung, Schlauheit; Lügner, schlauer, listiger Mensch' (Vas. 441); vgl. auch Solonisch alax, Negidalisch olok, Iamutisch olak, Orotschisch olok, Mandschurisch xolo (Vas. 441). Für die Entlehnungsrichtung gilt somit das oben Gesagte.

1 C̈ber das Verhāltnis tungusischer und ostjakischer Vokale hinsichtlich ihrer Quantität lässt sich anhand von vier Beispielen noch nichts Sicheres sagen. Auf diese Frage werde ich in spāteren Untersuchungen zurückkommen.

2 Bei Beurteilung der kakuminalen Aussprache des $l$ in einigen ostjakischen Dialekten schliesse ich mich der Ansicht von $K$. Rédei an: "Das $l$ ist in stimmhaft-stimmloser Korrelation mit dem $A$. Bei dem $l$ ist also die Kakuminalität eine irrelevante Eigenart ... (Nord-ostjakische Texte [Kazym-Dialekt] mit Skizze der Grammatik, Göttingen 1968, S. 12). 
Der kulturhistorische Hintergrund macht die Übernahme auch bei diesem Wort begreiflich: die Tungusen waren seit altersher in den magischen Künsten und im Schamanismus berühmt, ihr Appellativ 'Zauberpriester' ist bei einigen Nachbarn sogar zu ihrer nationalen Benennung geworden (vgl. A. J. Joki, "Tunguse" - "Pomback", Studie Serica Bernhard Karlgren dedicata, Copenhagen 1959, 123 ff.).

Wie erklärt sich aber die Abweichung des ostjakischen Anlautes vom evenkischen? Seine Vertretung in den ostjakischen Dialekten entspricht ja urostj. * $a$ (vgl. W. Steinitz, Geschichte des ostjakischen Vokalismus 57; im weiteren: OVok.). Hier müssen wir wohl mit einem Fall des Vokalwechsels rechnen, der von Steinitz schon in seiner Vokalgeschichte beschrieben wurde: "Bisweilen finden wir in den Entsprechungen ein und desselben Stammes in den verschiedenen Dialekten auf ${ }^{*} a \sim$ ${ }^{*} u$. . zurückgehende Vertretungen; z.B. ${ }^{*} a$ : V.-Vj. alam, Surg. åsəm, Irt. otam, Ni. Scher. otəm, Kaz. әләm, Ber. olam, Obd. alam 'Schlaf' ...*u: V.-Vj. ulam, Trj. usəm, Obd. uulam 'Traum'" (OVok. 59). Im Aufsatz "Einige Kapitel aus der obugrischen Vokalgeschichte» [Forschen und Wirken. Festschrift zur 150-Jahr-Feier der Humboldt-Universität zu Berlin Bd. III (1960)] stellte er dann für den Wechseltyp weitere Beispiele zusammen und zeigte zugleich, dass ostj. ${ }^{*} u \sim *^{*} a$ durchweg wog. ${ }^{*} \bar{u}$ entspricht (339 ff.); vgl. ausserdem id.: Zur Geschichte des ob-ugrischen Vokalismus, Zeitschr. Phon. 17 (1964), 639. Analog dazu dürfen wir - wie ich glaube - auch mit einer Entsprechung ev. ${ }^{*} u$ : ostj. ${ }^{*} u \sim{ }^{*} a$ rechnen und annehmen, dass im Ostjakischen dann die ${ }^{*} a$-Vertretung verallgemeinert wurde. Da das Wort in mehreren Dialekten, darunter in den archaischen Vach und Vasjugan fehlt, ist es im Ostjakischen vermutlich nicht besonders alt. Allerdings müssen wir mit einer Entlehnungszeit vor der Beendigung des Labialisierungsprozesses von $* a$ rechnen. Da diese Entwicklung in Irtysch um 1750 schon abgeschlossen gewesen ist (vgl. OVok. 57), muss die Übernahme - wegen der Konda- und Krasnojarskije-Belege - vor der Mitte des 18. Jahrhunderts erfolgt sein. Die Entsprechungen von $l$ und a bedürfen keiner weiteren Kommentare. Die Vertretung von ev. $k$ durch ostj. $x$ erinnert an eine Auslaut- 
regelung bei syrjänischen Lehnwörtern des Ostjakischen: „wenn die Entsprechung des Vokals vor einem Klusil ein Hintervokal ist, so ist die Entsprechung des Klusils velares $x$ bzw. kin (Toivonen: op. cit. 108).

4. Unser nächstes Wort ist ǔkśm (Ni. š Kaz.), NazT wakšam, Keu. ukśam, Vol. ýkcus, Ahl. uksim 'unrein (Frau bei der Menstruation und nach der Niederkunft; Keu. Ni.); widerlich (Vol. Ahl.), abscheulich, verflucht (Ahl.); etwas Garstiges (ঙัS KazSt.); unverschämt, schamlos in Reden; zänkisch, streitsüchtig (Kaz.)' (DEW 52).

Sein Original ist vermutlich ev. uksin 'Geschwür, Abszess, Eiterbeule' (Vas. 434). Die Wortfamilie ist im Tungusischen weitverbreitet: Negidalisch uktə 'faul, verfault, ungesund, schädlich'; Orotschisch uksi, Nanaisch uki 'Geschwür, Eiterbeule' (Vas. 434). Für die Entlehnungsrichtung gilt also auch hier das oben Gesagte.

Der Anlaut geht auf urostj. ${ }^{*} u$ zurück (OVok. 76). Die im Ostjakischen erfolgte Palatalisierung von $s$ ist als ein Zeichen der Expressivität aufzufassen. Der Wortinhalt berechtigt uns vollauf zu dieser Annahme, die expressive Palatalisierung ist für das Westostjakische charakteristisch (vgl. G. Ganschow, Affektiv-deskriptive Wortbildungssuffixe im Ostjakischen, Congr. Internat. Fenno-Ugristarum Budapestini hab., insbesondere 190; id., Die Verbalbildung im Ostjakischen 95; G. Sauer, Die Nominalbildung im Ostjakischen $127 \mathrm{f}$.). ə in der zweiten Silbe ist offenbar Ergebnis einer jüngeren Entwicklung, denn sowohl bei Vologodskij als auch bei Ahlqvist finden wir $i . m$ statt $n$ im Auslaut kommt auch bei syrjänischen Lehnwörtern des Ostjakischen vor (vgl. Toivonen, op. cit. 121) und dürfte keine Schwierigkeit für die Vergleichung bereiten.

Was die Wortbedeutung angeht, scheint sie sich im Ostjakischen zur abstrakteren hin weiterentwickelt zu haben - eine Erscheinung, die bei Lchnwörtern überall zu beobachten ist. Aus der Bedeutung 'Geschwür, usw.' konnte zuerst leicht die speziellere Bedeutung 'etwas Garstiges' (wie heute noch in Scherkal und Kazym) entstehen. Die eigene Sprache hatte ja für den durch das Fremdwort ursprünglich ausgedrückten In- 
halt einheimische Wörter reichlich bereit: K. F. Karjalainens Ostjakisches Wörterbuch zählt allein mit der Bedeutung 'Geschwür' acht Wörter auf, deren grösster Teil sicherlich alt ist. Gerade in solchen Situationen wird mit Vorliebe dem Lehnwort eine neue Funktion aufgetragen. Da die Grenzziehung zwischen Substantiv und Adjektiv in finnisch-ugrischen Sprachen, und erst recht im Obugrischen, nie streng durchgeführt wurde (die morphologische Kennzeichnung des Adjektivs ist in den obugrischen Sprachen heute noch nur ansatzweise der Fall), ist die adjektivische Erweiterung des Bedeutungfeldes als weiterer Schritt der Entwicklung durchaus normal.

5. Es scheint im Ostjakischen auch ältere Entlehnungen zu geben, nämlich Wörter, die in allen Dialektgebieten vorkommen. $\mathrm{Zu}$ ihnen gehört das letzte in diesem Beitrag zu bohandelnde Beispiel:

ăj3 (V Vart. Likr. Mj.), Vj. VK ăja, Trj. ăjз (ăjam), J ăjo (ăjam), Irt. ăj, DT auch ăj, Ni. Kaz. Pit. ŏj, š Sy. ŏj, O ăj, Reg. oj, aj, Ahl. oi 'Glück, Erfolg (z.B. bei Unternehmungen: 'Trj.; bei der Jagd: V Trj. Š); — Gut (Ahl.)'. Aus dem Ostjakischen wurde das Wort auch ins Wogulische übernommen: Szil. oj (DEW 20 f.).

Das ostjakische Wort kann gut verglichen werden mit ev. aja 'das Wohl, Gutes, gut, wohlwollend, lieb, usw.' (Vas. 44, Benz. 50, N. Poppe, Vergleichende Grammatik der Altaischen Sprachen I, Wiesbaden 1960,66 ). Das Wort ist im ganzen tungusischen Sprachgebiet bekannt: Solonisch, Negidalisch, Orotschisch, Orokisch, Udiheisch, Oltschaisch, Nanaisch aja, Solonisch, Lamutisch aj (Benz. 50, Vas. 44). Die rekonstruierte urtung. Form ist *aja (Benz. 50). Der Wortstamm hat in weiteren altaischen Sprachen Entsprechungen: Mongolisch aja 'Angemessenheit, Anstand, Entsprechung, günstiger Umstand', Kasakhisch aja 'best' (Entlehnung) (vgl. Poppe, op. cit. 66, vgl. auch G. J. Ramstedt, Einführung in die altaische Sprachwissenschaft I, Helsinki 1957, 97). Da einerseits der Stamm im Altaischen offenbar auf ein beträchtliches Alter zurückblickt, andererseits - zumindest nach unserem jetzigen Wissen - ausser im Ostjakischen (und Wogulischen als Lehnwort aus dem Ostjakischen) in keiner weiteren finnisch-ugrischen Sprache 
vorkommt, ist aja zweifellos im Ostjakischen ein Lehnwort, und nicht etwa umgekehrt. Als unmittelbare Lehngeber kommen dabei aus geographischen Gründen nur tungusische Dialekte in Frage, abgesehen davon, dass allein schon die beste semantische Übereinstimmung diese Entscheidung nahelegen würde.

Die lautliche Entsprechung der Formen ist so klar, dass sie keiner ausführlicher Kommentare bedarf. Der ostjakische Anlaut geht auf urostj. ${ }^{*} \breve{a}$ zurück (OVok. 81). Der Auslaut entspricht urostj. ${ }^{*} a$, wie das u.a. am Beispiel unseres Wortes von G.Sauer festgestellt wurde (Congr. Secund. Internat. Fenno-Ugristarum Pars I, 459).

6. Während an der Existenz tungusischer Lehnwörter im Ostjakischen m.E. kein Zweifel mehr besteht, ist die Frage, wo und unter welchen Begleitumständen die Übernahme erfolgte, noch weitgehend ungeklärt. Hier müssen wir uns vorläufig mit einigen Hinweisen begnügen.

Die jüngeren Lehnwörter dürften von den westlich des Jenissej lebenden Evenkiern übernommen worden sein. Es gibt nämlich zwei evenkische Sprachinseln in unmittelbarer Nähe del Ostjaken: die zum Nördlichen Dialekt (erbogotschenische Mundartgruppe) gehörende Ilimpiische Mundart, und die zum Südlichen Dialekt (katanggische Mundartgruppe) gehörende Symische Mundart (vgl. Benz. 10, Vas. 644f. und Dialektkarte). Beide Gruppen sind verhältnismässig junge Bewohner ihres Siedlungsgebietes. In russischen Archivmaterialien des 17. Jahrhunderts ist noch nirgends die Rede von ihnen; die Durchsicht des für diese Frage entscheidenden Werkes Б. О. Долгих, Родовой и племенной состав народов Сибири в XVII в., Москва 1960 verlief mit negativem Ergebnis. Für die heutige Lage sind die folgenden Stellen bei J. Benzing, Einführung in das Studium der Altaischen Philologie und der Turkologie, Wiesbaden 1953, aufschlussreich: „Die Ewenkier der nördlichen Gruppe sind erst verhältnismässig spät in die Jenisséische Taiga eingewandert, als sie durch die Jakuten aus dem ViljújFlussgebiet verdrängt wurden. Diese langsame Abwanderung von Osten, aus dem Wohngebiete der Jakuten nach Westen hin dauert heute noch an.» "Der symische Unterdialekt (nach 
dem Flusse Sym) erstreckt sich westlich rom Jenisséi bis zum Ob (in der Nähe der Stadt Narym) und darüber hinaus über den Vas'jugan und die Dem'janka bis gegen den Irtysch hin. Im Norden reicht er bis ins Flussgebiet des Eloguj, im Süden zum Culym, zur Ket' und zum Kas» (36). Dafür, dass Ostjaken tatsächlich Kontakte verschiedener Art zu diesen Evenkiern hatten, gibt es auch Belege. So berichtet M. A. Castrén an mehreren Stellen seiner "Reiseberichte und Briefe aus den Jahren 1845-1849" (Hrg. A. Schiefner), St. Petersburg 1856, über solche Begegnungen (s. u.a. $218 \mathrm{ff} ., 258$ ).

Hinsichtlich der älteren tungusisch-ostjakischen Kontakte sind wir vorläufig auf Vermutungen angewiesen. Eines scheint jedoch sicher zu sein: es gibt nichts, was g e g e n die Annahme der Berührungen sprechen könnte, vieles macht dagegen die Hypothese wahrscheinlich. "Es scheint, dass die R us sen crst während der Eroberung Sibiriens im 17. Jahrhundert mit den kleinen, scheuen Tungusenstämmen bekannt geworden sind, obgleich diese beweglichen Nomaden, Fischer und Jäger bisweilen sehr weit nach Westen - bis nach den nordwestlichen Teilen Russlands - gewandert waren." (A. J. Joki, op. cit. 123, Sperrung von mir.) Das Schweigen der älteren russischen Quellen hat also keine unsere Annahme entwertendr: Beweiskraft. Ausser russischen Quellen können wir aber --. leider -.- mit keinen geschriebenen Denkmälern rechnen. Doch es bleiben zwei Erkenntnisquellen übrig: die Sprache, deren Aussage gerade beim Fehlen historischer Dokumente schwer wiegt, und die mündliche Überlieferung. Die Befragung dẹ Sprache hat gerade erst begonnen. Was die Überlieferung angeht, so gibt es hier überraschende Mitteilungen, wic die folgende: "Etwas südlich von der Mündung des Kasym, in Tugijanskije, lebt eine ostjakische gens tunkas sir 'tungusische gens' (offizielle Name: Grischkin), deren Angehörige sich nach der Meinung der anderen Ostjaken von ihnen durch ihre dunkle Gesichtsfarbe unterscheiden. Offenbar handelt es sich um eine ostjakisierte tungusische Gruppe. Die nächsten 'Tungusen leben zwar ca. $500 \mathrm{~km}$ weiter östlich, es ist aber bekannt, dass kleine: nomadisierende tungusische Gruppen Wanderungen von vielen hundert Kilometern unternommen haben, wobei sie u.a., wie 
ich feststellen komnte, im Gebiet der südlichen Ostjaken auf Jagdzügen bis zum Irtysch gelangten.» (W. Steinitz, Totemismus bei den Ostjaken in Sibirien, Ethnos 1938, 127 f.) Derartige Angaben müssen natürlich nach Möglichkeit überprüft werden. Dass aber in Märchen, Sagen ı.ä. viel Wahres oft Jarhtausende hindurch aufbewahrt wird, gehört heutzutage zu den bekannten Tatsachen.

Istvíx Fu'taky 